\title{
Ocorrência de reação imediata generalizada à intradermorreação de Montenegro
}

\author{
Immediate and generalized reaction to \\ Montenegro skin test
}

\author{
Aline Fagundes ${ }^{1}$, Keyla Belizia Feldman Marzochi ${ }^{1}$ \\ e Mauro Celio de Almeida Marzochi ${ }^{1}$
}

\begin{abstract}
Resumo É descrito pela primeira vez um caso de reação exantemática imediata e generalizada após teste de Montenegro com antígeno mertiolatado. A reação consistiu de eritema urticariforme generalizado associado a prurido. O prurido desapareceu na primeira hora após a medicação com brometazina por via oral e a erupção 12 horas após.
\end{abstract}

Palavras-chaves: Teste de Montenegro. Leishmania. Reação sistêmica. Mertiolate.

\begin{abstract}
The authors describe for the first time a case of immediate and generalized reaction to Montenegro skin test with merthiolated antigen. This reaction consisted of generalized cutaneous rash with pruritus, and was treated with oral bromethazine. The pruritus disappeared an hour after medication and the rash 12 hours later.
\end{abstract}

Key-words: Montenegro skin Test. Leishmania. Systemic reaction Merthiolate.

Reações sistêmicas ocorrendo após testes intradérmicos são consideradas raras, embora casos fatais possam ocorrer ${ }^{5}{ }^{12}{ }^{13}$. Tais reações são mais associadas à realização de testes de alergia a antibióticos, particularmente penicilina ${ }^{13}$, porém mesmo o teste tuberculínico pode desencadeá-las ${ }^{1}$. Outros tipos de teste cutâneo, porém, podem desencadear reações sistêmicas de hipersensibilidade imediata ${ }^{9}$ e mesmo fenômenos naturais, como picadas de insetos, podem desencadear tais reações ${ }^{10}{ }^{11}$. No entanto, não são descritas reações severas após aplicação intradérmica de antígenos parasitários para diagnóstico. Durante um inquérito epidemiológico utilizando-se a Intradermorreação de Montenegro (IDRM) realizado na cidade de Manaus envolvendo 187 indivíduos, foi verificada a ocorrência de um caso de reação imediata generalizada dentro dos primeiros 30 minutos após a aplicação do teste intradérmico.

\section{RELATO DO CASO}

A reação ocorreu em um voluntário que havia recebido uma aplicação de antígeno de Montenegro (produzido com 40 microgramas de nitrogênio protéico/ml, diluído em salina mertiolatada a 1:10000 e outra aplicação concomitante da solução diluente do antígeno (salina mertiolatada a 1:10000).

A reação caracterizou-se por apresentação de prurido generalizado concomitante a erupção cutânea urticariforme, consistindo de placas eritematosas e sobrelevadas que se difundiram por todo o corpo, após 15 minutos da aplicação dos testes. Os locais dos testes, no entanto, permaneceram sem alterações. Não foi verificada a ocorrência de edema de glote, dispnéia, hipotensão arterial ou de outros sinais de hipersensibilidade.

Imediatamente, foi administrada prometazina (25mg) por via oral, com a remissão do prurido cerca de 10 minutos após a medicação. O desaparecimento completo da erupção cutânea ocorreu cerca de 12 horas

\footnotetext{
1. Centro de Referência Nacional para Diagnóstico da Leishmaniose Tegumentar do Serviço de Parasitologia do Instituto de Pesquisa Clínica Evandro Chagas da Fundação Oswaldo Cruz, Rio de Janeiro, RJ.

Endereço para correspondência: Dr. Mauro Célio de Almeida Marzochi. CRNDLT/Dept ${ }^{\circ}$ Parasitologia/FIOCRUZ. Av. Brasil 4365, 21045-900 Rio de Janeiro, RJ. Tel: 5521 2598-4266/4263/4264 ramal 141/107; Fax: 5521 2590-9988.

e-mail:crnleish@ipec.fiocruz.br

Recebido para publicação em 25/2/2003.

Aceito em 26/5/2003
} 
após a aplicação dos testes, tendo o voluntário permanecido sob medicação (brometazina, de 6 em 6 horas, VO) nas 72 horas seguintes.

O voluntário era sadio, com 24 anos, sem história de leishmanioses, natural de Manaus e morador há mais de dez anos na cidade. Referia ser portador de alergias diversas (a xarope, a baratas e a sulfa), representadas por rinite e urticária. Negava a utilização de mertiolate, e referia uso rotineiro de antiinflamatórios para contusões (sic). Não havia realizado testes intradérmicos anteriores, nem recebera qualquer vacina nos seis meses anteriores à realização do estudo.

Na leitura da IDRM 48 horas após a aplicação, o voluntário encontrava-se sem sinais e sintomas, em bom estado geral. As endurações apresentadas foram de $6 \mathrm{~mm}$ para a IDRM e de $9 \mathrm{~mm}$ para o teste controle com salina mertiolatada. As reações locais aos testes neste voluntário não diferiram morfologicamente das apresentadas por todos os outros voluntários sadios do estudo.

\section{DISCUSSÃO}

Não encontramos na literatura outros relatos de reação sistêmica após a realização do Teste de Montenegro. No entanto, o mertiolate (Timerosal) é capaz de provocar reações locais do tipo imediato quando utilizado por via mucosa ${ }^{6}$, ou do tipo retardado quando aplicado por via intradérmica ${ }^{378}$. Além disso, indivíduos com história de hipersensibilidade a determinados antiinflamatórios (p.ex piroxican) podem apresentar hipersensibilidade cruzada ao Timerosal ${ }^{2}$.

A história clínica do voluntário testado indica atopia, com hipersensibilidade generalizada. No entanto, não apresentava manifestações alérgicas de qualquer natureza no momento da realização das aplicações, e costumava utilizar antiinflamatórios sem queixas de manifestações alérgicas. Possivelmente, o Timerosal presente no antígeno para IDRM foi o responsável pela reação imediata ocorrida, tendo sido o voluntário orientado para não utilização de qualquer produto que inclua esta substância, sob qualquer forma e evite o uso de anti- inflamatórios.

Ressalta-se que o mesmo apresentou reação local morfologicamente típica de hipersensibilidade tardia na leitura de 48 horas após a aplicação dos testes, depois da total regressão dos sinais e sintomas de hipersensibilidade imediata e generalizada apresentados inicialmente, tendo sido considerado positivo ao teste de Montenegro.

Este caso ressalta a importância da cautela na aplicação de testes intradérmicos, mesmo em indivíduos saudáveis, da necessidade de uma minuciosa anamnese antes da aplicação dos mesmos e valorização da referência de alergias, dispondo-se sempre de tratamento à mão, bem como do estabelecimento de um sistema de vigilância de efeitos adversos para testes cutâneos. Indivíduos que desconhecem seu potencial de alergia ao mertiolate, ou com história compatível de atopia, devem receber vacinas ou testes intradérmicos com antígenos preservados com timerosal com muita cautela. Após aplicação de qualquer teste cutâneo, é recomendado observar o paciente por, pelo menos, 20 minutos $^{4}$.

Estudos estão em andamento visando a estabelecer o melhor diluente/ preservativo para o antígeno de Montenegro e para a padronização de leishmaninas livres de componentes inespecíficos.

\section{REFERÊNCIAS BIBLIOGRÁFICAS}

1. Froeschle JE, Ruben FL, Bloh AM. Immediate hypersensitivity reactions after use of tuberculin skin testing. Clinical Infectious Disease 34:E12-13, 2002

2. Ikezawa Z, Kitamura K, Osawa J, Hariya . Photosensitivity to piroxicam is induced by sensitization to thimerosal and thiosalicylate. Journal Investigative Dermatology 98:918-922, 1992.

3. Imperato PJ, Fofana B, Sow O, Diallo $S$,. Leishmanin skin sensitivity in the inland delta of the Niger. Tropical and Geographical Medicine 26:303-306, 1974.

4. Lockey RF. Adverse reactions associated with skin testing and immunotherapy. Allergy Proceedings 16: 293-296, 1995.

5. Lockey RF, Benedict LM, Turkeltaub PC, Bukantz SC. Fatalities from immunotherapy (IT) and skin testing (ST), Journal of Allergy Clinical Immunology 79: 660-677, 1988.

6. Maibach HI. False-positive intradermal skin tests and thimerosal. Jama 220: 126, 1972.

7. Maibach $\mathrm{H}$. Acute laryngeal obstruction presumed secondary to thiomersal (merthiolate) delayed hypersensitivity. Contact Dermatitis 1: 221-222, 1975.
8. Marzochi KB, Marzochi MA, Silva AF, Grativol N, Duarte R, Confort EM, Modabber F. Phase 1 study of an inactivated vaccine against American tegumentary leishmaniasis in normal volunteers in Brazil. Memórias do Instituto Oswaldo Cruz 93: 205-212, 1998.

9. Novembre E, Bernardini R, Bertini G, Massai G, Vierucci A. Skinprick-test-induced anaphylaxis. Allergy 50: 511-513, 1995.

10. Reisman RE, Osur SL. Allergic reactions following first insect sting exposure. Annales Allergy 59: 429-432, 1987.

11. Rhoades RB, Schafer WL, Schmid WH, Wubbena PF, Dozier RM, Townes AW, Wittig HJ. Hypersensitivity to the imported fire ant. A report of 49 cases, Journal of Allergy Clinical Immunology 56:84-93, 1975.

12. Valyasevi MA, Maddox DE, Li JT. Systemic reactions to allergy skin tests. Annales Allergy Asthma Immunology 83:132-136, 1999

13. Valyasevi MA, Van Dellen RG. Frequency of systematic reactions to penicillin skin tests. Annales Allergy Asthma Immunology 85:363-365, 2000. 